\title{
Aesthetics and Design for Group Music Improvisation
}

\author{
Mathias Funk, Bart Hengeveld, Joep Frens, and Matthias Rauterberg \\ Department of Industrial Design, Eindhoven University of Technology, \\ Den Dolech 2, 5612AZ Eindhoven, The Netherlands \\ \{m. funk, b.j.hengeveld,j.w. frens, g.w.m.rauterberg\} atue.nl
}

\begin{abstract}
Performing music as a group-improvised or from sheet music—is an intensive and immersive interaction activity that bears its own aesthetics. Players in such a setting are usually skilled in playing an instrument up to the level where they do not need to focus on the "operation" of the instrument, but can instead focus on higher-level feedback loops, e.g., between players in their section or the entire group. Novel technology can capitalize on these higherlevel feedback loops through the creation of interactive musical instruments that stimulate playing in groups (collaborative music rather than parallel music). However, making this experience accessible to fresh or novice players involves two challenges: how to design (1) musical instruments for such a setting and experience, and (2) instrument support that extends the interaction between players to their instruments. This allows to interact not only via their instrument with other human players, but directly with other instruments, producing a much richer and more intertwined musical experience. The paper shows results from a class of design students and reports on the lessons learned.
\end{abstract}

Keywords: Design, Interaction design, System design, Music, Improvisation, Co-creation.

\section{Introduction}

Group music performance is one of the oldest interaction settings dating back thousands of years in human history. While the musical instruments have certainly changed over time, the actual interaction between players has remained a constant factor, resulting in a direct, immersive and expressive experience that is joyful not only to the musicians, but also to the audience. Our current time allows for the creation of novel musical instruments once more, with the advance of embedded and networked technology. These allow us to take on another approach to group music where the instruments themselves can take on a more active role, resulting in a new expressive aesthetics.

In the design of aesthetic musical experiences as we approach it in this paper, three important notions are explained as preliminaries: (1) group improvisation as the activity that has the main focus in this paper, (2) extension of control as a necessary ingredient to new ways of improvisation, and (3) new musical instruments as the means to 
realize new musical improvisation experiences. These three are explained in the following.

\subsection{Group Improvisation}

In this paper, we focus on musical improvisation in group settings. In our view this activity involves multiple simultaneous feedback loops of musical expression [1] as shown in Figure 1. We see these loops as universal attributes of group music performance, whether played from sheet music (e.g., in a classical orchestra) or without (e.g., in a jazz quartet). We illustrate these loops first, before moving to the topic of group improvisation.

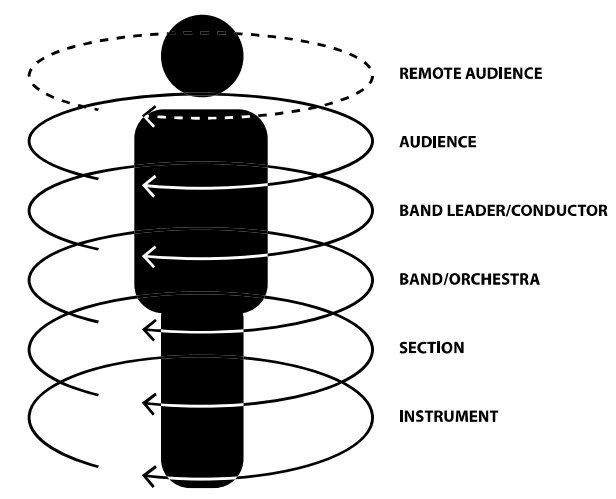

Fig. 1. Different layers of feedback loops in musical performance

Firstly, no matter the musical setting or situation, a musician interacts with his musical instrument. The musician plays the instrument, which in turn generates a sound, to which the musician can respond (e.g., in intonation or expression). This feedback loop is at the core of playing music.

Secondly, in any setting involving musical interaction (e.g., multiple musicians together or a single musician and a play-along CDs) a musician can belong to a section. For example, a drummer and a bass player both belong to a rhythm section. All musicians within a section have to sound well together, which adds a feedback loop to the aforementioned one.

The third feedback loop originates when sections start belonging to a larger musical body. For example, a cellist (feedback loop one) in a classical orchestra belongs to the cello section (feedback loop two), but also to the string section (consisting of the first and second violins, violas, cellos and basses, feedback loop three). Moreover, the string section belongs to an even larger body: the orchestra.

To keep all these sections musically aligned, orchestras have a conductor who imposes his interpretation on the orchestra. This adds a fourth feedback loop. In jazz bands the role of the conductor often moves from musician to musician within a single piece of music. 
The fifth feedback loop we identify is the one involving the audience: when the audience is enthusiastic typically musicians start performing better.

The sixth feedback loop we identify is less direct, but a feedback loop nonetheless: delayed feedback on your performance through sales, downloads, streams, Facebook likes, Tweets, etcetera. Clearly this feedback loop is of a different character than the previous five, but also one that may become more relevant in the near future.

As mentioned, we consider these feedback loops not unique for improvised group music, but pertaining to group music in general. What interests us in group improvisation though is the how the feedback loops are dependent on self-organization. Let us explain this.

Transcribed music has a clear organizing factor: the sheet music. Despite the openness for music interpretation or the relativity of tempi (allegro ma non troppo) or expressions (crescendo poco a poco) sheet music gives a common direction to all involved musicians. In improvised music much (not all) of this direction is absent; often music originates 'on the fly', depending on factors such as the group composition, the setting, the musical backgrounds of the group members, and more. This makes that a single musical design (e.g., a jazz standard) will sound differently each time.

From an interaction design perspective we find this highly interesting as it opens up a new domain of musical instruments in which technology-mediated, interactive musical instruments can lead to a new musical aesthetics. We elaborate on this in the next section. For example, we foresee that extending instrumental control among musicians (on which we elaborate in the next section) will lead to a different form of instrumentalism, as it will require musicians to accept other musicians to have an influence in the first feedback loop described in this section. As a consequence we anticipate that this will have repercussions on most of the other feedback loops, as shared control redefines the (self-) organizational structure between instrumentalists, sections etcetera. This will hopefully not only lead to a new aesthetics of instrumentalism alone, but also of group musicianship and of the resulting music.

\subsection{Extending Control}

In traditional group music settings, interaction happens usually non-verbal (to not disturb the music, or because one's voice would not carry over the music) using sign language, gestures, mimics, but mostly by listening to other instruments and watching other players play their instruments. Using this as an input, the players will adapt, for instance, volume, attenuation, and tempo of their own play-basically the application of multiple feedback loops as shown above. The connection between a player and other players' instruments is unidirectional in this process: the player can only perceive sound coming from another player's instrument, but not influence it-only indirectly, by influencing the other player. See Figure 2 (left side) for a visualization of this principle: players interact bi-directionally with their own instruments and with other players, and uni-directionally with other players' instruments.

What if a player could also influence the instruments of certain other players? As one can see from Figure 2 (right side), which shows a visualization of this new 
principle, the network of connections between players and instruments is denser and entirely bi-directional: players interact both with their own instruments, other players, and their instruments.
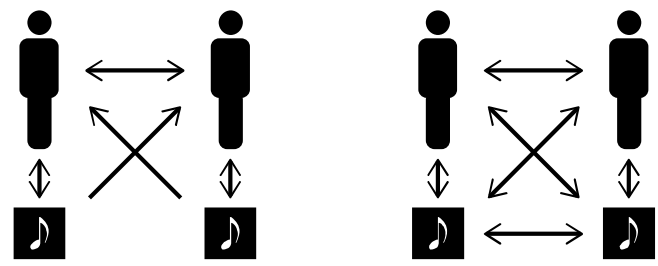

Fig. 2. Interaction modes in traditional music performance settings (left side) and in the proposed new setting (right side). Human players at the top interaction with each other, but also with their instruments denoted at the bottom of each figure.

This new interaction creates richer feedback loops, and necessarily adds to the complexity of the performance. In addition, this interaction mode changes how players perceive the setting of the performance: there is less a strong and exclusive bond between players and their own instruments. Instead, instruments become shared artifacts-same as the music has been already [2].

\subsection{New Musical Instruments}

Musical instruments have grown more sophisticated and nowadays allow for much richer expression. However, at the same time, the level of skills required to play state of the art musical instruments is higher than ever before. Some instruments cannot be played properly without years of (formal) training. Some instruments are too large or expensive to be owned and played by one's own will. Finally, nowadays frequent, ubiquitous, and high load music consumption has nurtured our tastes to a level where listening naïve musical expression or even just the sounds created by musical instruments are often not appreciated anymore-we simply do not enjoy the music we are able to create [3].

In the remainder of this paper, after showing related work, we will report on the process and outcomes of designing for group music improvisation, which is succeeded by important lessons learned. The paper concludes with a summary and an outlook on future steps of this line of design research.

\section{Related Work}

Although related work (interactive musical instruments designed specifically for group improvisation) is scarce, the history of interactive, electronic musical instruments in general is rich. Typically the Theremin and the Moog synthesizer are seen as pivotal milestones in this history, but recent work is superfluous and compelling. For example, Bevilacqua et al. explore digitally enhanced bow gestures in their "Augmented String Quartet" [4], IRCAM's MO project [5] offers a modular system 
allowing for the exploration of gestural interfaces for musical expressions, while other extend to mimics [6] or shift the interaction to collaborative objects [7], for instance, reacTable [8], which is a widely acclaimed interactive music tabletop. Another objective in this project was to open access to improvisation [9] as well as extending the control of musical instrument operation [10].

\section{Designing for Group Music Improvisation}

The challenges scoped in the introduction section are addressed in this section, describing the course of 5-month-long design project carried out by 20 Industrial Design undergraduate students. The project was initially framed as a system design project, but that alone would not properly capture the complexity: it is rather a setting, in which multiple designers collaboratively design a multi-user system consisting of multiple (radically) different and unique devices acting individually and as a whole. Designing for this new situation is easier said than done. A profound difficulty is that we seemingly cannot simply transfer our 'designing for interaction methods and tools' to this new paradigm. This has several reasons. Firstly, the systems we are aiming at are essentially too extensive and our targeted user-groups too heterogeneous to simply choose an approach (e.g., bottom-up or top-down) and start designing. Moreover, as designing for systems is relatively uncharted territory, we even don't know if these existing approaches apply or if we need an alternative hybrid approach [11]. Secondly, the interaction with systems appears to be different than with interactive standalone products as systems are more focused on facilitating opportunities for behavior rather than on disclosing functionalities.

The goal was a Group Music Improvisation System (GMIS) and students focused on different parts of the system, e.g. sound generation, sound modification, and technical infrastructure. We will first look at the process, how the designers approached the project and moved on to their own sub-projects, always with the GMIS in mind. Then, we will present the outcomes of this project, a number of instrument prototypes and the system as a whole.

\subsection{Setup and Process}

As described in the previous section we anticipated the requirement for a different design approach towards this project, for various reasons. To streamline the project as much as possible we stimulated all students to act as much as they could as a selforganizing system themselves, in order to have them co-shape the project and get grip on (musical) group dynamics. We organized weekly and bi-weekly coaching sessions, as well as regular jam sessions during which students could try out experiential mockups and prototypes of their designs together. In addition to these activities several workshops and invited talks were organized, for example involving a designer and maker of traditional musical instruments, and a composer and performer of contemporary electronic music. 
All students were stimulated to design in iterative cycles of designing, building and evaluating models and experiential prototypes of different levels of detail, using a process known as the Reflective Transformative Design Process (RTDP, [12]), with which they were all well-acquainted. One of the fortes of this process is that it is highly flexible and not prescriptive; in other words it allowed for exploring hybrid systems design approaches.

\subsection{Outcome}

The outcomes of the design project were plenty, about 16 devices or installations from 20 students, some of which were working in groups of four. As already mentioned in the process section, self-organization happened-although quite late in the process-in the form of multiple dimensions (as depicted in Figure 3).

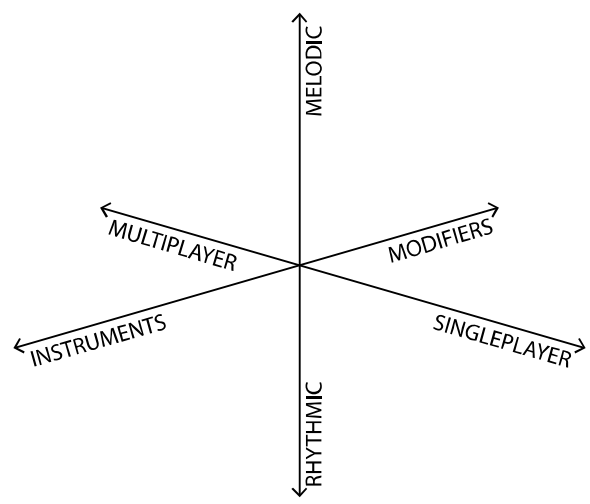

Fig. 3. Dimensions of outcomes as observed from the project: melodic-rhythmic (vertical), instruments-modifiers (left front to right back), multiplayer-single player (left back to right front).

Most importantly, the students divided into a large cluster creating music instruments, called generators, and another large cluster working on sound effects, called modifiers. The former mostly used tactile input captured by different kinds of sensors, mapped this to sound parameters, and finally emitted sound. The latter used, for instance, microphones and live sampling techniques to acquire sound input, which in turn could be processed and emitted again. Other modifiers used tangible interaction to shape the sound such as shown in Figure 4. The processing was determined by, again, tactile input. This dimension is shown in Figure 3 as one horizontal axis, which is crossed by the other two important dimensions about users (multiplayer vs. single player) and about musical attributes (melodic vs. rhythmic).

As shown in the introduction, this design project had also the objective to extend control and likewise enrich the feedback loops in action during a performance. The mapping of user and instrument is important, and the outcome showed that both single user instruments as well as collaborative multi-user instruments were possible and could contribute to a diverse and rich performance. 


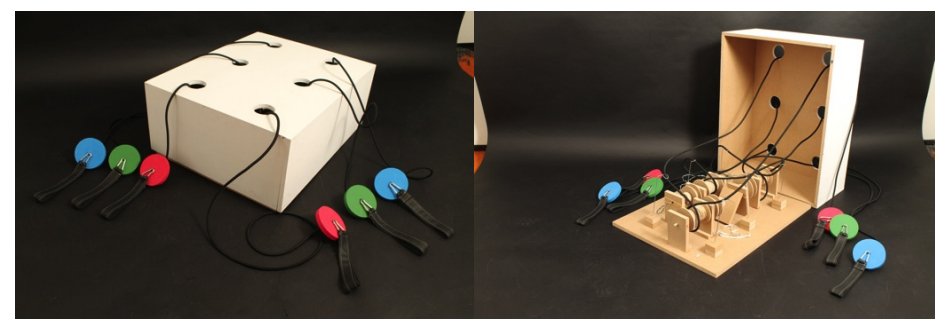

Fig. 4. Sound modifier, which uses tension on ropes attached to (or simply grabbed by) different players to modify sound. Ropes with same-colored handles have a mutual influence.

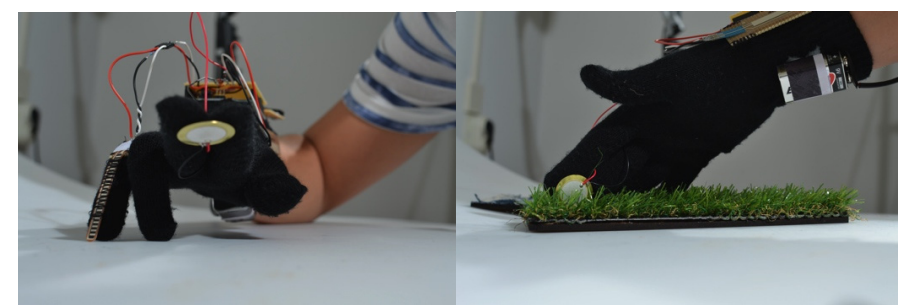

Fig. 5. Glove tangible sound generator. From textures sound is generated upon touch and stroking motions. Can even be played rhythmically.

The third dimension of outcome can be found in the content of produced sound and music, however naturally attributed more to sound generators than modifiers: some of the designers focused on interaction that produces melodic sound, aiming at pitch, tone, and tonal changes. The reason we distinguish this from more rhythmic instruments is that indeed most of the melodic instruments could be played with rhythmic accuracy and instead allowed players to work out aesthetic melodies or harmonics. The sounds produced by these instruments were, for instance, long tones, with slow transients and attack times, and long or undefined decays. Another example of focusing on the aesthetics of sounds was an instrument, with which the player could explore and play haptic textures of various materials (see Figure 5). On the other side of the spectrum, rhythmic instruments were usually very accurately playable, sometimes even purely acoustic instruments, sometimes relying on sampling and percussive sound generation.

\section{$4 \quad$ Results}

The results of this project can be seen from two angles: process and actual outcome. Both are worthwhile to report on and shed light on the overall task of designing for an aesthetic experience of interaction in a group performance.

The kind of design process students applied was no surprise, as they had learned and adopted a design process [12] that focuses on the individual designer with supplementary collaborative activities. This slowed them down in the first part of the 
design project. After a few weeks, we could however observe changing patterns: better communication and self-organization happened. Communication changed in terms of focus, from technology and prototyping, to what the students actually wanted to achieve in terms of music and experience. Self-organization at group level helped to focus on different aspects of the experience, such as sound generation and sound modification.

The process observed by us is very much characterized by rapid swings in terms of ideas, scopes, and technical foundations. As with any other explorative process, the swings are large in the beginning when most explorative action happens, and only over time processes stabilize until the point a narrow project is scoped and can be followed-through with minor changes. The problem arose when the design processes and swings of multiple designers were not synchronized and would not converge in the end. When reaching the final stage of the process, the processes should continue in parallel, which was what we had envisioned when setting up the design project. Unfortunately this could hardly be observed.

The outcomes of this design project were diverse and certainly product of a challenging process, however, the concepts and prototypes did not work as intended in a systematic way. We had aimed for truly connecting instruments and a lively jam session at the end, but the instruments did not work out that well due to (human) communication problems, which were pretty much unlike we have encountered before. Designing systems is a hard task, especially with multiple designers involved. The design students found it hard to focus on both micro and macro level, i.e. to design a musical instrument individually, and a system together. This required not only another perspective on design responsibility in the students, but also a certain acceptance of self-sacrifice: designing as a system demands individual designers to 'kill their darlings' - their part in the sum-for the sake of the whole. However, it is also clear that 16-20 different instruments, influencing each other would not only be a huge technical challenge, but also not produce the intended sonic results. A positive result from this design project is indeed that the designers interpreted the rules of the briefing such that also smaller, more effective teams were permitted. Thus, cluster of 2 to 4 designers emerged which jointly created instruments that could influence each other (at least conceptually) and that also produced sound complementary in pitch, tone, volume, rhythm, and texture-in short, a manageable experience both for the players and the audience.

\section{Conclusions}

Did this study reveal a new aesthetic experience of interaction in a group performance? Looking at the division we made in section 1.1 (aesthetics of instrumentalism, of group performance and of the resulting music) we have to conclude that we are not there yet: most of the results were similar to related work, which does not mean that this work is not aesthetic-on the contrary_but simply that we were looking for a novel aesthetics based on the effect of extended instrument control. The majority 
of resulting designs hinted at this, but not more than that, although we saw very interesting directions in force-fit combinations of modified acoustic instruments, fully digital instruments and (loop) modifiers. These seemed to generate an intriguing musical aesthetics, which is arguably not novel, but can be capitalized on in future studies.

We saw one prominent exception though. One of the students designed a very interesting set of instruments-one two-stringed tonal synthesizer and an augmented percussive instrument-in a 3D surround sound setting, where both instruments had their own behavior but were also open for one behavior modification by the other instrument. This meant that the percussive instrument could influence the pitch of the tonal instrument, and the tonal instrument the attack of the percussive instrument. The output of the tonal instrument was omnidirectional, but the percussive instrument moved around randomly in the 3D surround sound space, which added an intriguing additional feedback loop to those mentioned in Figure 1. Both instruments had a learning curve, which kept them interesting and challenging for the instrumentalists.

This set of instruments was used in two live, improvised performances by two of the design coaches, semi-professional musicians themselves (drummer and bassist). Their experience as traditional musicians helped un-steepening the learning curve of the instruments, but the diminished control over their instrument's musical contribution demanded a new musicianship: they were forced to search for their (individual and collaborative) aesthetic appreciation of their (individual and collaborative) music while performing. This made that the music itself played a more prominent role in directing the musicians, rather than the other way around.

As shown above in the results section, the design process taken by most of the students was not collaborative and converging enough to succeed. The students did not conceptualize via the aesthetics of interaction that a group music improvisation system should embody (including the sound and music it should product), but instead focused too much on actual instruments, technology and connectivity. In the coming iteration of this project, students need to be given more guidance and direction in the first part of their projects to gain the right momentum and understanding of the differences in this design process compared to design processes they have learned and applied in the past. This will be done by requiring all students to first think about the sound and music they would like their instruments to produce, instead of conceptualizing the instruments directly. These sound and interaction concepts will be documented and presented by means of video prototypes early in the design process.

The second aspect that needs revision is the technical infrastructure that supports the connectivity. In the coming iterations, all students can rely on a common infrastructure made of open-source building blocks that are not too challenging to program and still allow for the right amount of flexibility to design new instruments.

Acknowledgments. The authors would like to thank all students and coaches in the "Group Music Improvisation System" project that took place in the Out of Control theme at the Department of Industrial Design in Fall 2012. We thank Tom van ' $t$ Westeinde, Trieuvy Luu for allowing us to use their photographs. Special thanks to Vleer Doing for providing two new instruments and the infrastructure for influence. 


\section{References}

1. Bahn, C., Hahn, T., Trueman, D.: Physicality and feedback: a focus on the body in the performance of electronic music. In: Proceedings of the International Computer Music Conference, pp. 44-51 (2001)

2. Nacsa, J., Barakova, E., Frens, J.: Sharing meaning and physical activity through a tangible interactive lighting object. In: Procedings of the Second Conference on Creativity and Innovation in Design - DESIRE 2011, pp. 227-230. ACM Press, New York (2011)

3. Bryan-Kinns, N., Healey, P.: Decay in Collaborative Music Making. In: Schnell, N., Bevilacqua, F. (eds.) Interfaces, pp. 114-117. ACM Press, New York (2006)

4. Bevilacqua, F., Baschet, F., Lemouton, S.: The Augmented String Quartet: Experiments and Gesture Following. Journal of New Music Research 41, 103-119 (2012)

5. Rasamimanana, N., Bevilacqua, F., Schnell, N., Guedy, F., Flety, E., Maestracci, C., Zamborlin, B., Frechin, J.-L., Petrevski, U.: Modular musical objects towards embodied control of digital music. In: Proceedings of the Fifth International Conference on Tangible, Embedded, and Embodied Interaction, pp. 9-12. ACM, New York (2011)

6. Funk, M., Kuwabara, K., Lyons, M.J.: Sonification of Facial Actions for Musical Expression. Design, 127-131 (2005)

7. Heinz, S., Modhrain, S.O.: Designing a shareable musical TUI. Technology 26, 339-342 (2010)

8. Jordà, S., Geiger, G., Alonso, M., Kaltenbrunner, M.: The reacTable: exploring the synergy between live music performance and tabletop tangible interfaces. In: Proceedings of the 1st International Conference on Tangible and Embedded Interaction, pp. 139-146. ACM, New York (2007)

9. Hsu, W.: Design issues in interaction modeling for free improvisation. In: Parkinson, C., D'Arcangelo, G., Singer, E. (eds.) Proceedings of the 7th International Conference on New Interfaces for Musical Expression - NIME 2007, p. 367. ACM Press, New York (2007)

10. Cappelen, B.: Expanding the role of the instrument. In: Proceedings of the International Conference on New Interfaces for Musical Expression, pp. 511-514 (2011)

11. Hengeveld, B.J.: Designing LinguaBytes: A Tangible Language Learning System for Nonor Hardly Speaking Toddlers (2011)

12. Hummels, C., Frens, J.: The reflective transformative design process. In: Proceedings of the 27th International Conference Extended Abstracts on Human Factors in Computing Systems CHI EA 2009, pp. 2655-2658 (2009) 